\title{
Status of Semileptonic Hyperon Decays from Lattice QCD using 2+1 flavor Domain Wall Fermions
}

\author{
Shoichi Sasaki* \\ Department of Physics, Tohoku University, Sendai 980-8578, Japan \\ E-mail: Ssasakienucl.phys.tohoku.ac.jp
}

\begin{abstract}
We report the current status of hyperon vector couplings $f_{1}(0)$ in fully dynamical lattice QCD. The calculations are carried out with gauge configurations generated by the RBC and UKQCD collaborations with (2+1)-flavors of dynamical domain-wall fermions and the Iwasaki gauge action at two couplings, $\beta=2.13$ and 2.25. Our results show that signs of the second-order corrections of SU(3) breaking on the hyperon vector couplings in $\Sigma^{-} \rightarrow n l \bar{v}_{l}$ and $\Xi^{0} \rightarrow \Sigma^{+} l \bar{v}_{l}$ semileptonic decays are negative at simulated pion masses of $M_{\pi}=330-558 \mathrm{MeV}$. The size of the second-order corrections is comparable to what was observed in lattice calculations of kaon semileptonic decays. We also evaluate the systematic uncertainty on $f_{1}(0)$ due to the lattice discretization error.
\end{abstract}

31st International Symposium on Lattice Field Theory - LATTICE 2013

July 29 - August 3, 2013

Mainz, Germany

\footnotetext{
* Speaker.
} 


\section{Introduction}

Greater knowledge of the vector form factor $f_{1}$ in $\Delta S=1$ semileptonic hyperon decays paves the way for an alternative determination of the element $V_{u s}$ of the Cabibbo-Kobayashi-Maskawa $(\mathrm{CKM})$ matrix in addition to kaon semileptonic $\left(K_{l 3}\right)$ decays, leptonic decays of kaons and pions, and hadronic decays of $\tau$ leptons [1]. A stringent test of CKM unitarity through the first row relation $\left|V_{u d}\right|^{2}+\left|V_{u s}\right|^{2}+\left|V_{u b}\right|^{2}=1$ can be accomplished with the precision of $\left|V_{u s}\right|$ [1]. A theoretical estimation of the vector coupling $f_{1}(0)$, is required to extract $V_{u s}$ from the experimental rate of the hyperon beta decays [2] 3 .

In the iso-spin limit $\left(m_{u}=m_{d}\right)$, all $\Delta S=1$ semileptonic hyperon decays can be classified in four types of decays as $\Lambda \rightarrow N, \Sigma \rightarrow N, \Xi \rightarrow \Lambda$ and $\Xi \rightarrow \Sigma$. Their values of $f_{1}(0)$ are known to be equal to the SU(3) Clebsch-Gordan coefficients (see Table 1), up to the second-order in SU(3) breaking thanks to the Ademollo-Gatto theorem [4]. As the mass splittings among octet baryons are typically of the order of 10-15\%, an expected size of the second-order corrections is a few percent level. However, either the size or the sign of their corrections is somewhat controversial among various theoretical studies [3,5]. A model independent evaluation of SU(3)-breaking corrections is highly desired.

Recently, we have published the first result for the hyperon vector form factor $f_{1}$ for $\Xi \rightarrow \Sigma$ and $\Sigma \rightarrow N$ semileptonic decays from fully dynamical lattice QCD [5]. Our results show that the signs of the second-order corrections of SU(3) breaking on the hyperon vector couplings $f_{1}(0)$ are negative. It is consistent with what was reported in earlier quenched lattice studies [6] 7] and preliminary results from mixed action calculation [8] and dynamical improved Wilson fermion calculations [9]. We however recall that the tendency of the SU(3)-breaking corrections observed in lattice QCD simulations disagrees with prediction of covariant baryon chiral perturbation theory with the extended on-mass-shell (EOMS) renormalization scheme [10] as shown in Table 2

In Ref. [5], lattice calculations are carried out at a single lattice spacing, therefore the systematic error introduced by the lattice discretization was not estimated there. In this paper, we will report new results calculated at the finer lattice spacing and then evaluate the systematic uncertainty due to the finite lattice-spacing artifact.

\begin{tabular}{|ccc|}
\hline decays & $f_{1}(0)^{\mathrm{SU}(3)}$ & $\left(g_{1}(0) / f_{1}(0)\right)^{\mathrm{SU}(3)}$ \\
\hline$\Lambda \rightarrow N$ & $-\frac{\sqrt{6}}{2}$ & $F+\frac{1}{3} D$ \\
$\Sigma \rightarrow N$ & -1 & $F-D$ \\
$\Xi \rightarrow \Lambda$ & $\frac{\sqrt{6}}{2}$ & $F-\frac{1}{3} D$ \\
$\Xi \rightarrow \Sigma$ & 1 & $F+D$ \\
\hline
\end{tabular}

Table 1: The values of the vector coupling $f_{1}(0)$ and the axial-vector coupling $g_{1}(0)$ in four types of $\Delta S=1$ hyperon beta decays in the exact SU(3) symmetry world. The vector couplings are given by SU(3) ClebschGordan coefficients as $f_{1}(0)=f_{k l m}$, while the axial-vector couplings are governed by two parameters $F$ and $D$ as $g_{1}(0)=F f_{k l m}+D d_{k l m}$, where $d_{k l m}$ denotes the totally symmetric tensor of the SU(3) group. 


\begin{tabular}{|c|c|c|c|c||c|}
\hline \hline & \multicolumn{2}{|c|}{$\mathscr{O}\left(p^{3}\right)$ EOMS-CBChPT } & \multicolumn{2}{c||}{$\mathscr{O}\left(p^{4}\right)$ EOMS-CBChPT } & 2+1f DWF-LQCD \\
\hline & octet & + decuplet & octet & + decuplet & physical point \\
\hline$\Lambda \rightarrow N$ & -3.8 & -3.1 & $-3.6_{-0.9}^{+1.2}$ & $+0.1_{-1.0}^{+1.3}$ & - \\
$\Sigma \rightarrow N$ & -0.8 & -2.2 & $+3.9_{-2.8}^{+3.8}$ & $+8.7_{-3.1}^{+4.2}$ & $-3.0 \pm 1.1$ \\
$\Xi \rightarrow \Lambda$ & -2.9 & -2.9 & $-1.2_{-1.8}^{+2.4}$ & $+4.0_{-2.1}^{+2.8}$ & - \\
$\Xi \rightarrow \Sigma$ & -3.7 & -3.0 & $-1.3_{-0.2}^{+0.3}$ & $+1.7_{-1.6}^{+2.2}$ & $-2.7 \pm 0.7$ \\
\hline \hline
\end{tabular}

Table 2: $\mathrm{SU}(3)$-breaking corrections (in percentage), defined as $f_{1}(0) / f_{1}(0)^{\mathrm{SU}(3)}-1$, are obtained in covariant baryon chiral perturbation theory (CBChPT) [10] and (2+1)-flavor DWF dynamical lattice QCD [5]. Remark that the signs of the SU(3)-breaking corrections in CBChPT are basically changed between the $\mathscr{O}\left(p^{3}\right)$ and $\mathscr{O}\left(p^{4}\right)$ results.

\section{Numerical Results}

We use the RBC and UKQCD collaboration ensembles, which are generated at two lattice spacings, $a=0.114 \mathrm{fm}$ (coarse) and $a=0.086 \mathrm{fm}$ (fine), with two light degenerate quarks and a single flavor heavier quark and the Iwasaki gauge action [11] 12]. Their lattice sizes, $24^{3} \times$ 64 and $32^{3} \times 64$, correspond to almost the same physical volumes, where the spatial extent is approximately $2.7 \mathrm{fm}$ in physical units. The dynamical light and strange quarks are described by DWF actions with fifth dimensional extent $L_{s}=16$ and the domain-wall height of $M_{5}=1.8$ for these ensembles. We choose values of the light quark masses, $a m_{u d}=0.005,0.01$ and, 0.02 for the $24^{3}$ ensembles and $a m_{u d}=0.008$ for the $32^{3}$ ensembles in this study. The strange dynamical quark masses chosen as $a m_{s}=0.04$ for the $24^{3}$ ensembles and $a m_{s}=0.03$ for the $32^{3}$ ensembles are both close to the physical value. Details of simulation parameters with DWF gauge configurations are summarized in Table 3

The general form of the baryon matrix element for semileptonic decays $B \rightarrow b l \bar{v}_{l}$, is composed of the vector and aixal-vector transitions, $\left\langle b\left(p^{\prime}\right)\left|V_{\alpha}(x)+A_{\alpha}(x)\right| B(p)\right\rangle$, which are described by six form factors: the vector $\left(f_{1}\right)$, weak magnetism $\left(f_{2}\right)$, and induced scalar $\left(f_{3}\right)$ form factors for the vector current, and the axial-vector $\left(g_{1}\right)$, weak electricity $\left(g_{2}\right)$, and induced pseudo-scalar $\left(g_{3}\right)$ form factors for the axial current [2]. In this paper, we focus on the vector part of the baryon matrix element:

$$
\left\langle b\left(p^{\prime}\right)\left|V_{\alpha}(x)\right| B(p)\right\rangle=\bar{u}_{B}\left(p^{\prime}\right)\left(\gamma_{\alpha} f_{1}^{B \rightarrow b}\left(q^{2}\right)+\sigma_{\alpha \beta} q_{\beta} \frac{f_{2}^{B \rightarrow b}\left(q^{2}\right)}{M_{B}+M_{b}}+i q_{\alpha} \frac{f_{3}^{B \rightarrow b}\left(q^{2}\right)}{M_{B}+M_{b}}\right) u_{B}(p) e^{i q \cdot x},
$$

which are here given in the Euclidean metric convention (see Ref. [7] for details). The vector current is defined as $V_{\alpha}(x)=\bar{u}(x) \gamma_{\alpha} s(x)$ for $\Delta S=1$ decays. For convenience in numerical calculations, instead of the vector form factor $f_{1}\left(q^{2}\right)$, we consider the so-called scalar form factor

$$
f_{S}^{B \rightarrow b}\left(q^{2}\right)=f_{1}^{B \rightarrow b}\left(q^{2}\right)+\frac{q^{2}}{M_{B}^{2}-M_{b}^{2}} f_{3}^{B \rightarrow b}\left(q^{2}\right),
$$

where $f_{3}$ represents the second-class form factor, which is identically zero in the exact $\mathrm{SU}(3)$ limit [13]. The renormalized value of $f_{S}\left(q^{2}\right)$ at $q_{\max }^{2}=-\left(M_{B}-M_{b}\right)^{2}<0$ can be precisely evaluated by the double ratio method proposed in Ref. [6], where all relevant three-point functions are 


\begin{tabular}{|ccc|cccccc|}
\hline$\beta$ & $a^{-1}[\mathrm{GeV}]$ & $a m_{u d}$ & $N_{\text {conf }}$ & MD range & $N_{\text {sep }}$ & $N_{\text {meas }}$ & $t_{\text {src }}$ positions & $M_{\pi}[\mathrm{GeV}]$ \\
\hline 2.13 & $1.73(4)$ & 0.005 & 240 & $940-5720$ & 20 & 2 & 0,32 & $0.3297(7)$ \\
& & 0.01 & 120 & $5060-7440$ & 20 & 2 & 0,32 & $0.4200(12)$ \\
& & 0.02 & 80 & $1890-3470$ & 20 & 4 & $0,16,32,48$ & $0.5580(11)$ \\
2.25 & $2.28(3)$ & 0.008 & 100 & $980-2960$ & 20 & 2 & 0,32 & $0.3925(17)$ \\
\hline
\end{tabular}

Table 3: Summary of simulation parameters: the number of gauge configurations, the range, where measurements were made, in molecular-dynamics (MD) time, the number of trajectory separation between each measured configuration, the number of measurements, and source locations on each configuration. The table also lists the pion masses [11,12].

determined at zero three-momentum transfer $|\mathbf{q}|=0$. For the three-point functions, we use the sequential source method. Details of the construction of the three-point functions from the sequential quark propagator are described in Ref. [14].

Here we note that the absolute value of the renormalized $f_{S}\left(q_{\max }^{2}\right)$ is exactly unity in the flavor SU(3) symmetric limit, where $f_{S}\left(q_{\max }^{2}\right)$ becomes $f_{1}(0)$, for the hyperon decays considered here. Thus, the deviation from unity in $\left|f_{S}\left(q_{\max }^{2}\right)\right|$ is attributed to three types of the $\mathrm{SU}(3)$ breaking effect: (1) the recoil correction $\left(q_{\max }^{2} \neq 0\right)$ stemming from the mass difference of $B$ and $b$ states, (2) the presence of the second-class form factor $f_{3}\left(q^{2}\right)$, and (3) the deviation from unity in the renormalized $f_{1}(0)$. Taking the limit of zero four-momentum transfer of $f_{S}\left(q^{2}\right)$ can separate the third effect from the others, since the scalar form factor at $q^{2}=0, f_{S}(0)$, is identical to $f_{1}(0)$. Indeed, our main target is to measure the third one.

The scalar form factor $f_{S}\left(q^{2}\right)$ at $q^{2}>0$ is also calculable with nonzero three-momentum transfer $(|\mathbf{q}| \neq 0)$. To avoid unnecessary repetition, we simply give a reference [7], where all the technical details are available. We use the four lowest nonzero momenta: $\mathbf{q}=2 \pi / L \times(1,0,0),(1,1,0)$, $(1,1,1)$, and $(2,0,0)$. We then can make the $q^{2}$ interpolation of $f_{S}\left(q^{2}\right)$ to $q^{2}=0$ by the values of $f_{S}\left(q^{2}\right)$ at $q^{2}>0$ together with the precisely measured value of $f_{S}\left(q^{2}\right)$ at $q^{2}=q_{\max }^{2}<0$ from the double ratio. For the $q^{2}$ interpolation, the choice of the interpolation form does not affect the interpolated value $f_{S}(0)$ significantly. We simply prefer to use the values obtained from the monopole fit and quote a systematic error due to the $q^{2}$ interpolation as a difference between results obtained from the monopole and quadratic forms [5, 7] in the following discussion.

In Fig. 11 we first show the results of $\tilde{f}_{1}(0)=f_{1}(0) / f_{1}(0)^{\mathrm{SU}(3)}$ (filled circles) obtained from the $24^{3}$ ensembles as a function of the pion mass squared for $\Xi \rightarrow \Sigma$ (left panel) and $\Sigma \rightarrow N$ (right panel). In order to estimate $\tilde{f}_{1}(0)$ at the physical point, we perform a global fit of the data on $\tilde{f}_{1}(0)$ as multiple functions of $M_{K}^{2}-M_{\pi}^{2}$ and $M_{K}^{2}+M_{\pi}^{2}$

$$
\tilde{f}_{1}(0)=C_{0}+\left(C_{1}+C_{2} \cdot\left(M_{K}^{2}+M_{\pi}^{2}\right)\right) \cdot\left(M_{K}^{2}-M_{\pi}^{2}\right)^{2},
$$

which form is motivated by the Ademollo-Gatto theorem [5]. Here, we remark that our simulations are performed with a strange quark mass slightly heavier than the physical mass. To take into account this slight deviation in this global analysis of the chiral extrapolation, we simply evaluate a correction using the Gell-Mann-Oakes-Renner relation for the pion and kaon masses, which corresponds to the quark mass dependence of pseudo-scalar meson masses at the leading order of ChPT. Dashed and solid curves appeared in Fig. 1 1 represent the fitting results for the physical 

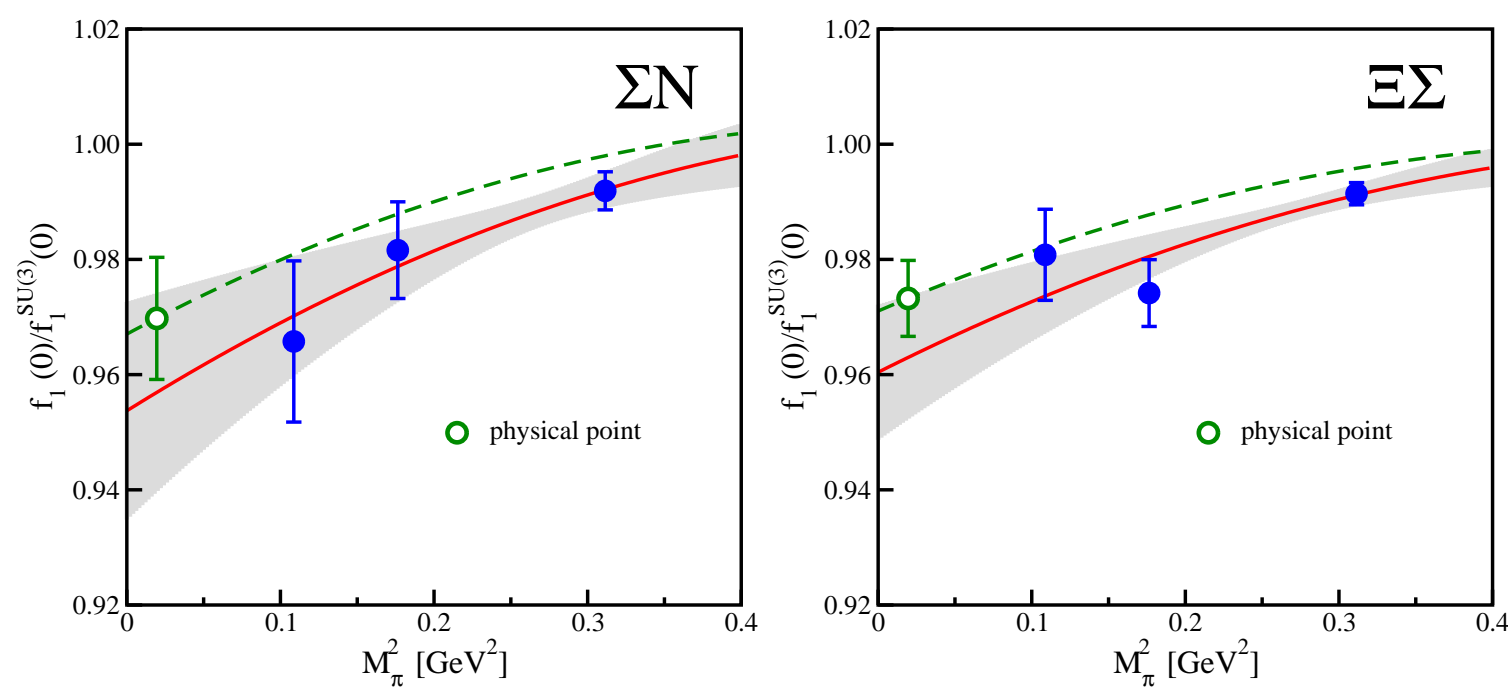

Figure 1: Chiral extrapolation of $\tilde{f}_{1}(0)$ for $\Sigma \rightarrow N$ (left) and $\Xi \rightarrow \Sigma$ (right), whose data calculated on the $24^{3}$ ensembles. Fitting curves indicated by dashed and solid curves represent the fitting results for the physical strange mass and the simulated one $\left(a m_{u d}=0.04\right)$, respectively.

strange mass and the simulated one $\left(a m_{s}=0.04\right)$, respectively. The extrapolated results of $\tilde{f}_{1}(0)$ at the physical point are denoted as open circles. It is worth emphasizing that the signs of the second-order corrections on $f_{1}(0)$ calculated in fully dynamical lattice QCD are negative in both $\Sigma \rightarrow N$ and $\Xi \rightarrow \Sigma$ decays. The tendency of the SU(3) breaking correction observed here disagrees with predictions of the latest baryon chiral perturbation theory result [10]. However, in Ref. [5], lattice calculations are carried out at a single lattice spacing. The systematic error introduced by the lattice discretization has not yet estimated there.

In this study, we additionally carry out lattice simulations with the $32^{3}$ ensembles, where the lattice spacing is finer than that of the $24^{3}$ ensembles. New data points are included in Fig 2 for checking the scaling behavior. The corresponding pion mass of the new data points is close to the second lightest quark mass in the $24^{3}$ ensembles. Regardless of the fact that the strange quark masses on two ensembles are slightly off each other, we find good scaling behavior in Fig. 2 The new data points obtained from the $32^{3}$ ensembles are consistent with the dotted curves, which are fit results only on data points of the $24^{3}$ ensembles. It indicates that the systematic error due to the finite lattice spacing is smaller than the statistical error.

Combined with the new data calculated at the finer lattice spacing, we finally quote our results of the renormalized $f_{1}(0)$ calculated from fully dynamical lattice QCD as

$$
f_{1}(0)= \begin{cases}-0.9698(106)_{\text {stat. }}(15)_{q^{2}}(36)_{m_{q}}(28)_{a^{2}} & \text { for } \Sigma \rightarrow N \\ +0.9732(66))_{\text {stat. }}(7)_{q^{2}}(5)_{m_{q}}(40)_{a^{2}} & \text { for } \Xi \rightarrow \Sigma,\end{cases}
$$

which the first errors ares statistical, and the second, the third and the fourth ones are estimates of the systematic errors due to our choice of $q^{2}$ interpolation, the reliability of the extrapolation to the physical point and the finite lattice-spacing artifact. The size of the second-order corrections observed here is comparable to what was observed in (2+1)-flavor DWF calculations of $K_{l 3}$ decays [15, 16. 

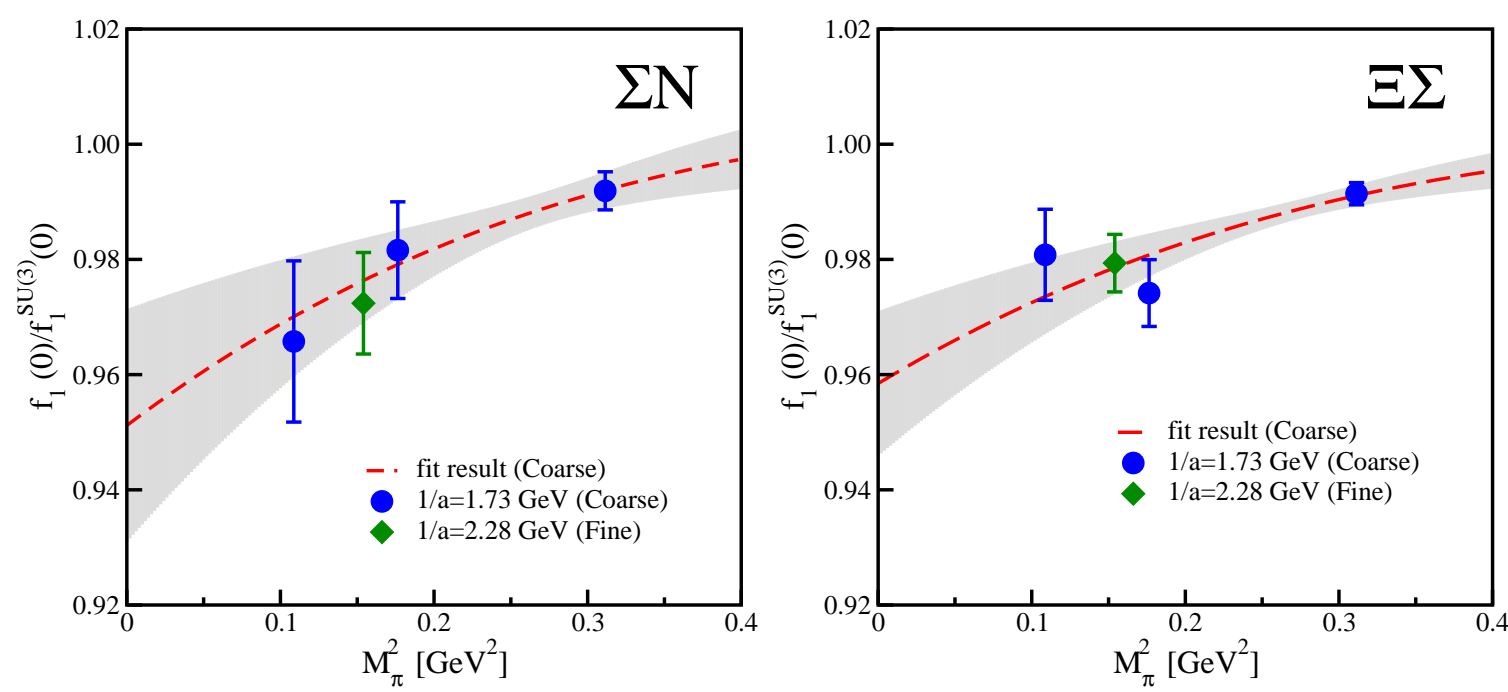

Figure 2: Comparison between the results calculated on the coarser (filled circles) and finer (filled diamonds) lattices.

\section{Summary}

We have studied the flavor SU(3)-breaking effect on the hyperon vector couplings $f_{1}(0)$ for the $\Xi^{0} \rightarrow \Sigma^{+}$and $\Sigma^{-} \rightarrow n$ semileptonic decays in (2+1)-flavor QCD using domain wall quarks at two lattice spacings. We have observed that the second-order corrections on $f_{1}(0)$ are negative for both $\Sigma \rightarrow N$ and $\Xi \rightarrow \Sigma$ decays at simulated pion masses of $M_{\pi}=330-558 \mathrm{MeV}$. We estimate the systematic uncertainty due to the lattice discretization error by the observed discrepancy between the results calculated at two lattice spacings. Recall that the systematic error due to the finite size effect was found to be negligibly small in the vector coupling of the beta decay [17]. The observed discrepancy between ours and the baryon ChPT results can not be accounted by the systematic errors in lattice QCD simulations, rather suggests that the baryon ChPT has a serious convergence problem as described in Table 2 .

We plan to increase the reliance on the chiral extrapolation using the $32^{3}$ ensembles at the lighter quark masses $\left(a m_{u d}=0.004\right.$ and 0.006$)$, which are closer to the physical point, and also to extend our research to calculate the axial-vector coupling $g_{1}(0)$ and also the weak electricity $g_{2}(0)$ in hyperon beta decays.

\section{Acknowledgments}

This work is supported by the JSPS Grants-in-Aid for Scientific Research (C) (No. 23540284). Numerical calculations reported here were carried out on KEK supercomputer system, the T2K supercomputer at CCS, University of Tsukuba, and also RIKEN Integrated Cluster of Clusters (RICC) facility. 


\section{References}

[1] E. Blucher and W. J. Marciano, " $V_{u d}, V_{u s}$, Cabibbo Angle, and CKM Unitarity," in J. Beringer et al. (Particle Data Group), Phys. Rev. D 86, 010001 (2012).

[2] For a review of hyperon beta decays, see N. Cabibbo, E. C. Swallow and R. Winston, Ann. Rev. Nucl. Part. Sci. 53, 39 (2003) and references therein.

[3] V. Mateu and A. Pich, JHEP 0510, 041 (2005).

[4] M. Ademollo and R. Gatto, Phys. Rev. Lett. 13, 264 (1964).

[5] S. Sasaki, Phys. Rev. D 86, 114502 (2012).

[6] D. Guadagnoli, V. Lubicz, M. Papinutto and S. Simula, Nucl. Phys. B 761, 63 (2007).

[7] S. Sasaki and T. Yamazaki, Phys. Rev. D 79, 074508 (2009).

[8] H. W. Lin, Nucl. Phys. Proc. Suppl. 187, 200 (2009).

[9] M. Gockeler et al. [QCDSF Collaboration and UKQCD Collaboration], PoS LATTICE2010, 165 (2010).

[10] L. S. Geng, J. Martin Camalich and M. J. Vicente Vacas, Phys. Rev. D 79, 094022 (2009).

[11] C. Allton et al. [RBC-UKQCD Collaboration], Phys. Rev. D 78, 114509 (2008).

[12] Y. Aoki et al. [RBC and UKQCD Collaborations], Phys. Rev. D 83, 074508 (2011) [arXiv:1011.0892 [hep-lat]].

[13] S. Weinberg, Phys. Rev. 112, 1375 (1958).

[14] S. Sasaki, K. Orginos, S. Ohta and T. Blum, [RIKEN-BNL-Columbia-KEK Collaboration], Phys. Rev. D 68, 054509 (2003).

[15] P. A. Boyle et al. [RBC+UKQCD Collaboration], Phys. Rev. Lett. 100, 141601 (2008).

[16] P. A. Boyle, J. M. Flynn, N. Garron, A. Jüttner, C. T. Sachrajda, K. Sivalingam and J. M. Zanotti, JHEP 1308, 132 (2013).

[17] T. Yamazaki et al. [RBC+UKQCD Collaboration], Phys. Rev. D 79, 114505 (2009). 